livraisons

d'Histoire

de l'Architecture

\section{Livraisons de l'histoire de l'architecture}

$18 \mid 2009$

D'architectures

\title{
Les architectes idéistes
}

The 'ideiste architects'

Die sogenannten ideistischen Architekten

\section{Marie-Astrid Pourchet}

\section{(2) OpenEdition}

\section{Journals}

Édition électronique

URL : http://journals.openedition.org/lha/229

DOI : 10.4000/lha.229

ISSN : 1960-5994

Éditeur

Association Livraisons d'histoire de l'architecture - LHA

Édition imprimée

Date de publication : 10 décembre 2009

Pagination : 105-114

ISSN : $1627-4970$

Référence électronique

Marie-Astrid Pourchet, "Les architectes idéistes », Livraisons de l'histoire de l'architecture [En ligne], 18 | 2009, mis en ligne le 10 décembre 2011, consulté le 06 mai 2019. URL : http:// journals.openedition.org/lha/229; DOI : 10.4000//ha.229

Ce document a été généré automatiquement le 6 mai 2019.

Tous droits réservés à l'Association LHA 


\title{
Les architectes idéistes
}

\author{
The 'ideiste architects' \\ Die sogenannten ideistischen Architekten
}

Marie-Astrid Pourchet

1 Trois architectes de la fin du XIX siècle et du début du $\mathrm{XX}^{\mathrm{e}}$ siècle sont dénommés « architectes idéistes » : François Garas (1866-1925), Gabriel Guillemonat (1866-1945) et Henri Provensal (1868-1934). Cette qualification permet de les identifier mais ils ne forment pas un véritable groupe: seule la similitude de leur parcours et de leur état d'esprit semble pouvoir les réunir. Le terme «idéiste» leur a été adjoint par Caroline Mathieu, conservateur en chef au musée d'Orsay, en référence au célèbre article d'Albert Aurier «Le Symbolisme en peinture - Paul Gauguin », publié dans le Mercure de France en février 1891. La définition d'une œuvre donnée par Aurier s'applique en premier lieu à la peinture de Gauguin, mais peut également convenir aux projets d'architecture de François Garas, Gabriel Guillemonat et Henri Provensal.

\section{Les débuts prometteurs de trois architectes}

2 Tous trois suivent leur formation à l'École des beaux-arts dont ils sont diplômés entre 1893 et 1898. François Garas est l'élève du très autoritaire Paul Blondel (1847-1897), connu pour avoir été l'architecte du Louvre, des Tuileries et de la Cour de cassation. Guillemonat a pour professeur Jean-Louis Pascal (1837-1920), prix de Rome en 1866 et fervent opposant à l'éclectisme, avec lequel il entretient des relations de confiance comme le suggèrent les missives retrouvées dans son dossier d'élève à l'École des beaux-arts. Quant à Henry Provensal, il est inscrit dans l'atelier de deux professeurs : Jean-Henry Duray et Julien Guadet (1834-1908), auteur d'éléments et théorie de l'architecture, livre de référence des étudiants en architecture pendant de nombreuses décennies.

3 La formation suivie par les trois jeunes hommes se révèle ancrée dans le classicisme: Pascal et Guadet s'étaient illustrés par leur opposition véhémente au projet de réforme de l'École des beaux-arts mené par Viollet-le-Duc en 1863. Cette formation se double toutefois d'un penchant pour l'éclectisme, né pour une part à l'école des beaux-arts où il 
s'est décliné en plusieurs tendances. L'éclectisme englobe toute une série de courants, de l'historicisme au régionalisme, ce dernier bénéficiant de l'appui de l'École des beaux-arts qui y perçoit le moyen de renouveler le répertoire formel ${ }^{1}$. Cela peut expliquer en partie le choix esthétique des premiers projets présentés par les trois architectes au Salon de la société nationale des beaux-arts (SNBA) (ill. 1).

III. 1 : « L'Asyle de rêve. Façade », Henri Provensal,

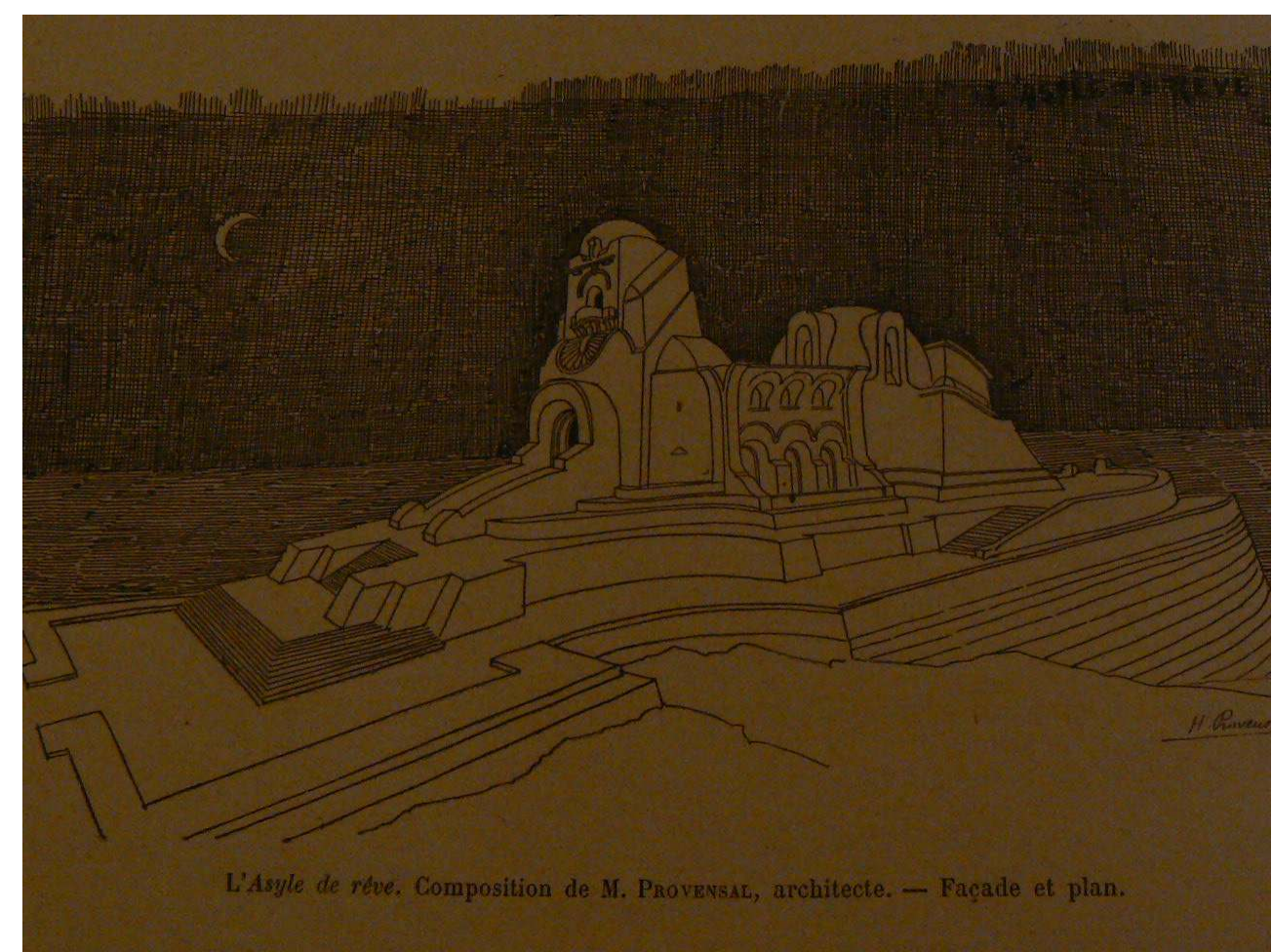

L'Architecture, 9e année, n50, samedi 12 décembre 1896, pages 376-378

Cl. Marie-Astrid Pourchet.

4 Les trois jeunes architectes exposent régulièrement dans ce Salon à partir de 1893. La société nationale des beaux-arts se veut en rupture avec le salon des artistes français et devient l'un des évènements mondains français qui attire l'élite internationale des arts : artistes, mécènes et collectionneurs. La participation à un tel évènement est donc une reconnaissance en soi pour Garas, Guillemonat et Provensal. Cependant, la section d'architecture est la plus restreinte et attire moins le public, aussi cette reconnaissance demeure limitée au monde professionnel et critique de l'architecture. Ces architectes ne seront donc pas connus du grand public. Une véritable mise en lumière de ces trois jeunes architectes apparaît davantage à travers l'exposition Impressions d'architectes organisée à la galerie Le Barc de Bouteville. L'ensemble des projets d'architecture exposés lors de ces deux types de manifestation constitue l'unique corpus parvenu jusqu'à nous. Il est d'autant plus important pour l'étude de ces trois architectes qu'aucun de ces projets ne fut réalisé. 


\section{La quête d'un idéal}

5 Ces projets d'architecture exposés à la société nationale des beaux-arts ou lors de l'exposition Impressions d'architectes ne résultent en effet d'aucune commande : ils sont le fruit des recherches de Garas, Guillemonat et Provensal. Ceux-ci tentent d'élaborer une nouvelle architecture résultant de leurs intérêts pour les divers arts que sont la musique, la littérature, la poésie... Ce renouvellement de l'art architectural est élaboré à partir de certaines idées transmises par les grands artistes de l'époque, notamment le musicien Richard Wagner (1813-1883), dont l'œuvre influence les arts, dépassant le domaine de la musique. Son influence sur l'architecture peut paraitre étonnante mais Richard Wagner n'est-il pas l'un des premiers à concevoir la notion d'un « art total »? En effet, ce n'est pas tant sa musique que ses écrits dont nous parlons, car il y explique la recherche du Gesamtkunstwerk, l'idéal du chef d'œuvre d'art total qui réunirait les différents arts. Les trois architectes ont sûrement pris connaissance de l'œuvre et des théories de Wagner puisque la découverte de Wagner en France remonte à la génération de Gérard de Nerval et Théophile Gautier. Son œuvre ne cesse d'être diffusée en France, puis, après sa mort, est fondée en 1885 la Revue wagnérienne qui se fait l'organe privilégié de cette diffusion. Garas, Guillemonat et Provensal ont donc certainement pu avoir connaissance des idées de Wagner et s'y sensibiliser. L'objectif de cette notion « art total » véhiculée par Richard Wagner ne réside pas en l'unique union des arts mais bien en l'élévation de l'âme, réalisée à travers la moralisation de l'art et le retour à la spiritualité.

6 Cette quête de spiritualité est notamment accomplie, dans le domaine de la peinture, par Gustave Moreau (1826-1898) et Puvis de Chavannes (1824-1898). Gustave Moreau peint des personnages qui sont souvent issus des grands mythes grecs et de l'histoire sainte, au sein d'une architecture surchargée de décors qui pourrait être assimilée à certains projets de François Garas tel celui du Temple à la Pensée. Quant à Pierre Puvis de Chavannes, artiste de renommée internationale, son œuvre est marquée par l'idéalisme de l'école lyonnaise. Nous le percevons à travers des références à l'âge d'or ou bien à travers l'expression de l'équilibre entre la nature et l'homme. Garas, Guillemonat et Provensal ne pouvaient pas méconnaître l'œuvre de Puvis de Chavannes comme le certifie la présentation par Guillemonat et Provensal de projets de Monument à Pierre Puvis de Chavannes au salon de la société nationale des beaux-arts.

7 Les trois architectes font référence à de nombreux artistes au seine de leurs œuvres car ils sont à la recherche d'une architecture "savante ", multipliant les références envers tous les arts. Pour de concevoir cette nouvelle architecture, Garas, Guillemonat et Provensal entrent en opposition avec les principes de l'École des beaux-arts. Ils sont soutenus par l'architecte Frantz Jourdain (1847-1935) qui dénonce l'enseignement de l'École des beaux-arts et souhaite renouveler l'architecture. Frantz Jourdain apporte son soutien aux jeunes architectes et rédige la préface au catalogue de l'exposition Impressions d'architectes ${ }^{2}$.

8 L'étude des projets d'architecture parvenus jusqu'à nous, c'est-à-dire ceux qui sont conservés au musée d'Orsay, conjuguée à celle des écrits de Garas (Mes Temples ${ }^{3}$, Un Palais de la musique $e^{4}$, Temple à la pensée $e^{5}$ ) et de ceux de Provensal (L'Art de demain, vers l'harmonie intégrale ${ }^{6}$ nous permet de cerner au mieux leurs conceptions architecturales.

9 La fin du XIX siècle est marquée par la floraison de progrès techniques qui « révolutionne » la vie courante et le monde de l'industrie. Cette rapidité du progrès, loin 
des romans visionnaires de Jules Verne, n'est pas sans inquiéter certaines personnes, dont font partie les trois architectes idéistes. Si ces architectes font preuve d'une certaine réticence face aux profonds changements entrainés par les évolutions techniques ils ne rejettent pas pour autant les bienfaits de celles-ci : ainsi Garas projette d'employer le fer et le ciment armé pour construire le Temple à la Pensée, mais la foi totale que certains prêchent envers la science n'est pas partagée par les trois jeunes hommes. Provensal avance ainsi que « les progrès actuels accomplis par la science [...] n'ont nullement accru la félicité humaine $»^{7}$.

10 Les trois architectes accordent une place centrale à la pensée et à l'idéal ; ils espèrent réconcilier la science avec les grandes religions. Cette réconciliation peut s'effectuer notamment à travers l'art qui donne la traduction matérielle de concepts, idéologiques, voire spirituels. Garas, Provensal et Guillemonat les envisagent comme ne pouvant être qu'universels et éternels. Il s'agit, à travers leur art, de s'adresser au plus grand nombre de personnes. Ils adoptent ainsi une vision de l'art entièrement dépourvue d'élitisme, mais non de hauteur de pensée car l'un des rôles de l'art est d'élever l'âme de son spectateur. Les trois architectes se penchent par conséquence sur l'étude des monuments religieux, qui semblent les plus adaptés à l'élévation spirituelle, avec une prédilection pour le temple : « Depuis les origines du monde jusqu'à la Renaissance, on peut s'assurer que les monuments religieux sont les seuls endroits où se soit extériorisée l'âme humaine, au cours des siècles. Pénétrons donc dans ces lieux où l'homme a soulevé le voile du mystère. Demandons-leur de nous livrer le secret que tous recèlent au plus profond du sanctuaire ; c'est par une connaissance approfondie des différentes stases de l'humanité que nous parviendrons, nous élevant au-dessus des conceptions philosophiques auxquelles ces monuments ont offert une réalité plastique, à reconstituer une base solide aux édifices où puisse de nouveau s'extériorisée «la conscience des peuples ${ }^{8}$ ». Ces architectes accordent d'autant plus d'importance au temple qu'il s'agit du lieu où l'homme «essaiera de délivrer son moi du fardeau qui l'obsède et que les religions l'aidèrent à supporter ${ }^{9}$ ». La définition que donnent les architectes « idéistes » du temple ne se restreint pas à la définition d'un édifice religieux. Provensal qualifie ainsi la mairie de temple civil. Cet édifice est en effet le témoin de la naissance, du mariage et de la mort de chaque individu ${ }^{10}$. Cette conception est alors sans doute à l'origine de son projet d'une Mairie pour une ville de 3000 habitants (1897). Ces architectes accordent une grande importance au sentiment spirituel, quelle que soit sa nature et quelle que soit la religion (ill. 2). 


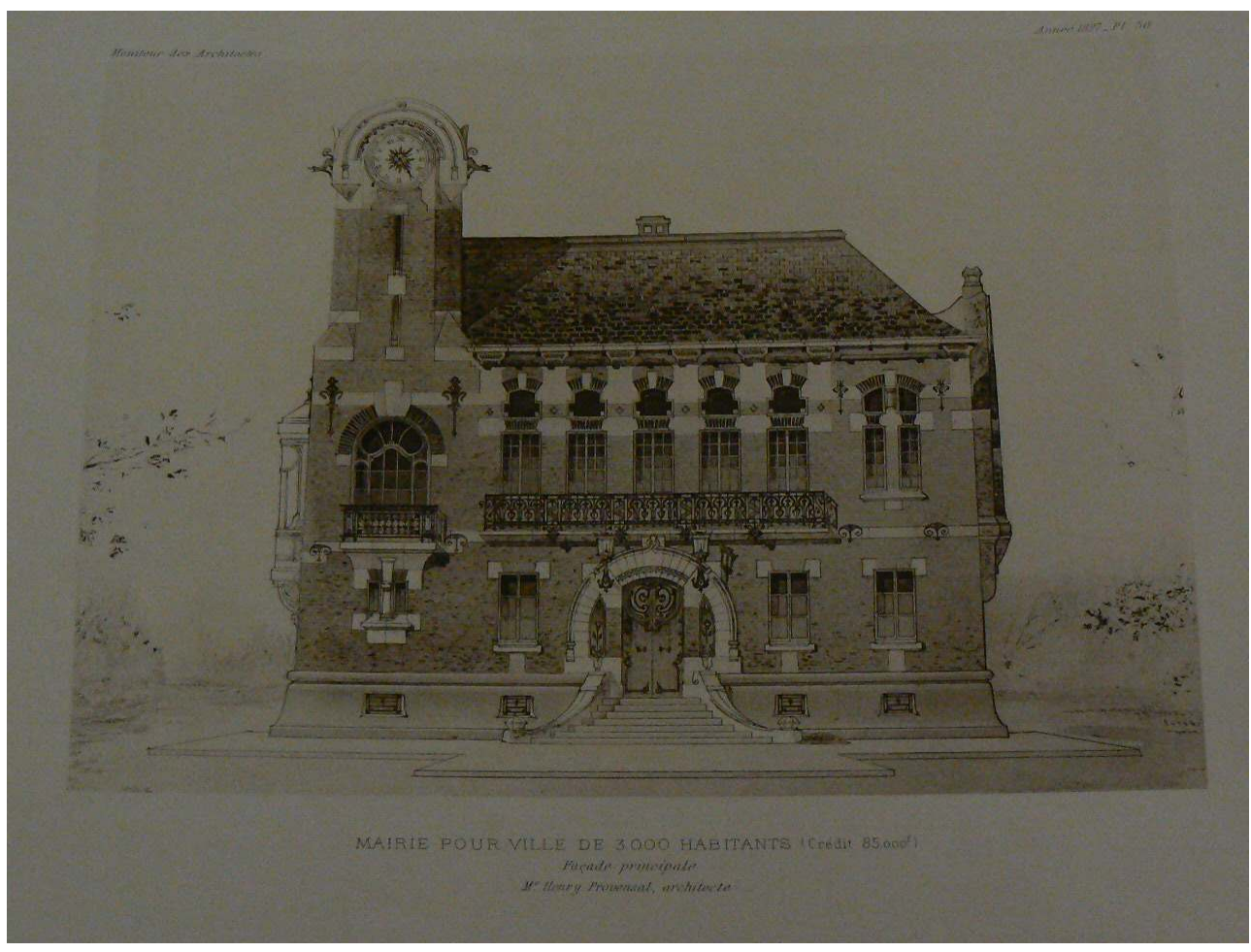

Le Moniteur des Architectes, Paris, septembre 1897, planche 50

Cl. Marie-Astrid Pourchet.

11 Les trois architectes ne font d'ailleurs jamais mention d'aucune religion spécifique. Leurs projets semblent résulter aussi d'influences diverses et pas uniquement classiques. Le Temple à la Pensée de François Garas devait ainsi comprendre dans sa décoration intérieure « les figures colossales des philosophies et des religions synthétisées par le calme éternel du Bouddha antique $»^{11}$.

\section{Un rapprochement avec le symbolisme}

L'ensemble de ces recherches rapproche alors les trois architectes du mouvement symboliste. Le terme de mouvement et non celui d'école est employé car aucune doctrine symboliste n'a été clairement établie. Le symbolisme est avant tout littéraire: sa naissance officielle peut être datée de 1886 en raison de la publication de plusieurs ouvrages. C'est ainsi qu'en 1886, sont publiés dans le numéro de janvier de la Revue wagnérienne des sonnets en hommage à Richard Wagner signés par Mallarmé, Verlaine, René Ghil, Stuart Merrill, Charles Morice, Charles Vignier, Teodor de Wyzewa et Édouard Jardin. Cet hommage au grand musicien prend toute son importance parce qu'elle constitue la première manifestation collective symboliste. En 1886 sont également publiées les Illuminations de Rimbaud, or le symbolisme se définit en partie par le contrecoup de la réception des écrits du jeune poète. Certains éléments de la « doctrine » symboliste apparaissent dans le Traité du verbe de René Ghil ainsi que dans le « manifeste » du symbolisme que Jean Moréas écrit dans Le Figaro du 18 septembre 1886. Le contenu de ce "manifeste » est assez vague mais est perçu comme l'acte de naissance du symbolisme en raison de la résonance qu'il a eu. Moréas expose tout de même un 
programme et une définition : le symbolisme « cherche à vêtir l'Idée d'une forme sensible qui, néanmoins, ne serait pas son but à elle-même, mais qui, tout en servant à exprimer l'Idée, demeurait sujette. L'Idée, à son tour, ne doit point se laisser voir privée des somptueuses simarres des analogies extérieures; car le caractère essentiel de l'art symbolique consiste à ne jamais aller jusqu'à la concentration de l'Idée en soi ${ }^{12}$. »

En 1886, la dernière exposition impressionniste est organisée : aussi est-ce également une année déterminante pour les beaux-arts car le mouvement symboliste qui débute en littérature va s'imposer dans les arts plastiques et les orienter vers de nouvelles recherches. Charles Morice publie en 1889 le très important ouvrage La Littérature de toute à l'heure dans lequel il précise que l'art symboliste doit être synthétique c'est-à-dire qu'il doit parler à n'importe quel individu et de tout, en rapprochant la musique, la peinture et la poésie. Cette opinion révèle un premier point de rapprochement avec les conceptions des trois architectes Garas, Guillemonat et Provensal. Il est fortement probable que les trois jeunes architectes aient lu Charles Morice ou bien aient eu vent de ses théories car le mouvement symboliste n'est pas apprécié instantanément par le public : il faut attendre l'année 1888 pour observer la véritable apparition d'un public symboliste. Cette date correspond plus ou moins à celles de l'entrée des trois jeunes hommes à l'École des beauxarts. Leurs années d'études sont d'ailleurs ponctuées de nombreuses publications symbolistes. Il est vrai que, dans ses débuts, le symbolisme est essentiellement littéraire mais il est également représenté par des œuvres d'art dont l'une des plus célèbres est $L a$ Vision après le sermon ou La Lutte de Jacob avec l'ange de Paul Gauguin (1888, National Gallery of Scotland, Édimbourg) qui représente une vision intérieure plus qu'une réalité ainsi que l'organisation d'un nouvel espace pictural. Il s'ensuit en 1889 une exposition organisée au café Volpini au cours de laquelle un " groupe symboliste et synthétique » se constitue. Les membres de ce groupe sont très divers tout autant que leurs styles, aussi la constitution d'une école est-elle difficile. Ces différents artistes partagent néanmoins certains principes clairement définis tels que le refus du monde contemporain et du réalisme, l'affirmation de son propre univers élaboré grâce à une vision intérieure, la prééminence de l'« idée » et de l' "abstraction ». Le texte d'Albert Aurier écrit à propos de Gauguin résume en quelque sorte les recherches menées par les différents artistes « symbolistes » lorsqu'il tente de donner une définition à l'œuvre d'art ${ }^{13}$ :

«L'œuvre d'art, telle qu'il m'a plu la logiquement évoquer, sera:

$1^{\circ}$ idéiste, puisque son idéal unique sera l'expression de l'Idée ;

$2^{\circ}$ symboliste, puisqu'elle exprimera cette Idée par des formes ;

$3^{\circ}$ synthétique, puisqu'elle écrira ces formes, ces signes, selon un mode de compréhension générale ;

$4^{\circ}$ subjective, puisque l'objet n'y sera jamais considéré en tant qu'objet, mais en tant que signe d'idée perçu par le sujet ;

$5^{\circ}$ (C'est une conséquence) décorative - car la peinture décorative proprement dite, telle que l'on comprise les Égyptiens, très probablement les Grecs et les Primitifs, n'est rien autre qu'une manifestation d'art à la fois subjectif, synthétique, symboliste et idéiste.»

Les visions d'architecture de Garas, Guillemonat et Provensal se rattachent-elles réellement au symbolisme? Est-il possible d'appliquer au domaine de l'architecture la définition d'œuvre d'art qu'Aurier donne de la peinture, et plus précisément à l'architecture conçue par ces trois architectes? L'importance de l' «Idée » dans le processus créatif est indéniable chez eux, comme le démontrent leurs écrits. Leur objectif consiste également à atteindre le plus grand nombre à travers l'usage de "symboles " compréhensibles par tous. C'est pourquoi leurs projets d'architecture se veulent riches en 
décor aussi adoptent-ils un certain aspect décoratif. La démonstration d'Aurier peut donc s'appliquer aux trois « architectes idéistes ».

Ce texte n'est pas la seule preuve du lien qui existe entre les « architectes idéistes » et le mouvement symboliste. Quelques caractéristiques du symbolisme peuvent être mise en avant pour mieux comprendre les liens entre le symbolisme et l'œuvre des trois architectes. Une de celles-ci est le recours aux mythes et légendes: des paysages évoquant les époques idéales de l'Âge d'or ornent les piliers du Temple à la Pensée conçu par Garas et Provensal fait appel à la figure sphinx pour son Asyle de rêve. Les artistes se rattachant aux recherches symbolistes font également référence au Moyen Âge, du moins à la vision dont ils ont hérité des recherches préraphaélites. Une trace de celles-ci apparait dans plusieurs projets de Garas, comme Intérieur d'artiste ou la Maison de retraite dans la montagne pour un artiste musicien et peintre (ill. 3).

III. 3 : « Maison de retraite pour un artiste, au Mont-Pilat, Cévennes, Loire, Façade latérale », François Garas

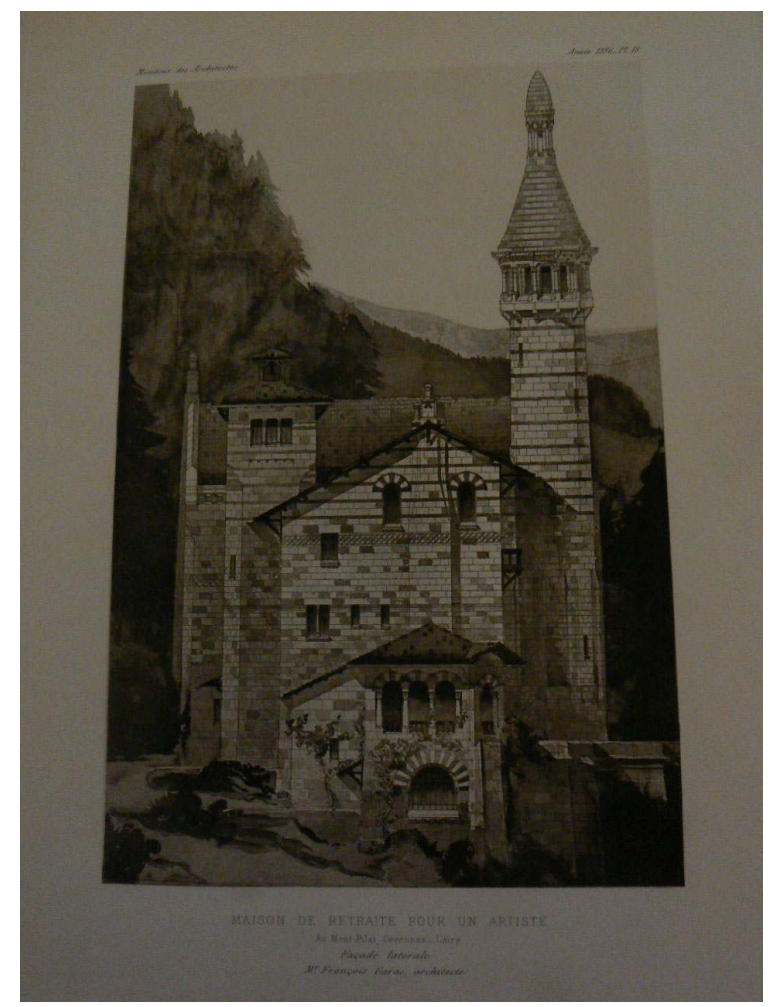

Le Moniteur des architectes, Angers, imprimerie Burdin et Cie, 1896

Cl. Marie-Astrid Pourchet.

Dans le premier, la période médiévale est présente dans l'aménagement intérieur de la pièce qui s'apparente plus à une vision qu'à une reconstitution archéologique; dans le second projet, l'appel au passé médiéval est réalisé à travers l'architecture de l'édifice qui est davantage proche de la réalité scientifique. Il s'agit en effet d'un des premiers projets de l'architecte qui est encore sous l'influence historiciste.

Pourtant ces références aux mondes passés et perdus ne relèvent pas de la nostalgie, elles participent plutôt à la critique de la société contemporaine et même à son rejet, ce qui a pour effet de tourner les artistes vers la recherche d'un sens profond du monde. Elles 
s'expriment à travers un goût prononcé pour le sacré voire l'adhésion au mysticisme qui les rapproche de la Rose-Croix.

Le rejet de la société industrielle n'est tout de même pas total car les architectes ne rejettent pas les progrès qu'elle entraîne : ils s'en font les défenseurs comme nous l'avons déjà précisé en incluant dans leurs projets différentes techniques nouvelles mais aussi en rendant gloire à l'industrie comme a pu le faire Garas avec son Palais de l'Industrie en 1911.

Si les artistes qualifiés de symbolistes se montrent aussi critiques envers la société contemporaine, c'est sans doute en raison de la référence qu'ils accordent au domaine du « sacré » qui nourrit bon nombre de leurs œuvres: Carlos Schwabe peint La Vierge des Lys (Amsterdam, musée Van Gogh) ; la même année, Auguste Rodin réalise la Main de Dieu (1898, Philadelphie, The Rodin Museum) après que Paul Gauguin a consacré à la figure du Christ une peinture emblématique (Le Christ jaune, 1889, Paris, musée d'Orsay). Garas, Provensal et Guillemonat sont dans le même état d'esprit et établissent un discours architectural imprégné de la notion d'une "Vérité » nourrie de la foi. Henri Provensal le théorise dans un article de L'Architecture ${ }^{14}$ où il prétend que : «L'art de l'avenir [...] aura pour objet de manifester la plus haute conscience religieuse des générations futures. On ne considérera comme art, dans l'avenir, que ce qui exprimera des sentiments poussant les hommes à l'union fraternelle ou encore, des sentiments assez universels pour être éprouvés par l'ensemble des hommes. » Ces rapprochements permettent ainsi de tisser un lien entre le mouvement symboliste, dans ses aspects littéraires et artistiques, et les " architectes idéistes » encore que les trois architectes n'ont pu y adhéré de manière institutionnelle ou associative. Ils semblent approuver, au vu de leurs projets d'architecture et de leurs écrits, les divers objectifs de celui-ci. Ils représentent ainsi l'une des rares applications des visions symbolistes à l'architecture. Cette rareté peut s'expliquer, entre autres, par les difficultés concrètes à donner vie à de telles architectures: si les architectes ont songé aux matériaux qui rendent possibles de telles constructions, ils se sont sans aucun doute désintéressés de la question financière. Leurs projets sont certes réussis au niveau artistique et suscitent des louanges de la part de certains critiques lors de leurs expositions sans avoir pour autant trouver un acquéreur. Les projets «idéistes» souffrent de ce manque de concrétisation car, malgré leur apparition dans quelques revues, ils restent peu diffusés, ce qui peut expliquer la méconnaissance dans laquelle ils demeurent aujourd'hui. Cette situation s'explique également par le découragement de Garas, Guillemonat et Provensal. Ils finissent en effet par abandonner leurs idéaux: François Garas se retire du métier d'architecte pour prendre la direction de la fabrique familiale. Gabriel Guillemonat et Henri Provensal poursuivent leur carrière mais se détournent de leurs projets pour concevoir de tout autres types d'architecture. Guillemonat se dirige vers une carrière plus académique en prenant la fonction d'architecte diocésain dans le Loiret avant de devenir professeur à l'école Boulle ainsi qu'architecte des palais du Louvre, des Tuileries et de l'Académie de médecine. Provensal, après l'obtention du deuxième prix au concours de la Fondation Rothschild, se concentre sur la construction d'habitations à bon marché (H.B.M). Il demeure tout de même un théoricien, n'abandonne pas ses idéaux et théorise ce type de construction dans son ouvrage L'Habitation salubre à bon marché $e^{15}$. 


\section{NOTES}

1. . Françoise Chaslin, « Le style urbain à la mode de Bretagne », Architecture, n 18, octobre 1980, $64 \mathrm{p}$.

2. . Charles Lucas, «Salmis d'expositions ", La Construction moderne, t.1896-1897, 12 année, $\mathrm{n}^{\circ} 11$, 12 décembre 1896, $630 \mathrm{p}$.

3. . François Garas, Mes Temples, Paris, impr. Michalon, 1907, 24 p.

4. . François Garas, « Un palais de la musique », L’Art décoratif, n², février 1908, 40 p.

5. . François Garas, Temple à la pensée : ma petite maison/Salon de la société nationale des beaux-arts, Paris, salon de la société nationale des Beaux-Arts, 1909, 11p.

6. . Henri Provensal, L'Art de demain, vers l'harmonie intégrale, Paris, Perrin, 1904, 322 p.

7.. Ibid

8. . Henry Provensal, L'Art de demain, op.cit.

9. . Henry Provensal, L'Art de demain, op.cit.

10. . Henry Provensal, L'Art de demain, op.cit.

11. . François Garas, Mes Temples, op.cit.

12. . Jean Moréas, "Un Manifeste littéraire », Le Figaro littéraire, $12^{\mathrm{e}}$ année, $\mathrm{n}^{\circ} 38$, samedi 18 septembre 1886, $4 \mathrm{p}$.

13. . Albert Aurier, «Le Symbolisme en peinture - Paul Gauguin », Mercure de France, t. II, année 1891, février 1891, $385 \mathrm{p}$.

14. . Henri Provensal, «L'Art nouveau », L’Architecture, $13^{e}$ année, $n^{\circ} 51$, samedi 22 décembre 1900, $503 \mathrm{p}$.

15. Henri Provensal, L'Habitation salubre à bon marché, Paris, Ch.Schmid, 1908, 80 p.

\section{RÉSUMÉS}

Trois architectes de la fin du XIX siècle et du début du XX ${ }^{\mathrm{e}}$ siècle sont dénommés « architectes idéistes »: François Garas (1866-1925), Gabriel Guillemonat (1866-1945) et Henri Provensal (1868-1934). Ils ne forment pas un véritable groupe mais cette qualification permet de les identifier : ils semblent pouvoir être réunis par la similitude de leurs parcours et de leur état d'esprit. Tous trois suivent en effet leur formation artistique à l'École des beaux-arts puis exposent au sein de la galerie Le Barc de Bouteville en 1896 et régulièrement au sein du Salon de la société nationale des beaux-arts (SNBA). Lors de ces différentes manifestations, ces trois architectes présentent essentiellement des projets d'architecture pour lesquels ils n'ont pas de commanditaire : fait important car leurs projets ne seront jamais réalisés. Leurs architectures sont cependant remarquées par les grands critiques de l'époque. Elles résultent de la volonté de renouveler, voire de créer une nouvelle architecture. Leur intérêt pour la musique, la poésie et la littérature les rapproche d'un courant artistique majeur de l'époque : le mouvement symboliste. Nous parlons de mouvement et non pas d'école car aucune doctrine symboliste n'a été clairement établie. Garas, Guillemonat et Provensal ont réalisé par leurs projets d'architecture la trop rare 
application des réflexions symbolistes. Ils font référence aux artistes de ce mouvement, avec lesquels ils partagent le même état d'esprit, ainsi qu'aux artistes appréciés des symbolistes.

At the end of the $19^{\text {th }}$ century and the beginning of the $20^{\text {th }}$ century, the three architects François Garas (1866-1925), Gabriel Guillemonat (1866-1945) and Henri Provensal (1868-1934) were termed 'ideiste architects'. Although they did not form a group, the term "ideistes architects'allows them to be considered as such due to the similarities in their approach and the way their styles developed. All three were students of the École des beaux-arts before showing their work together in the gallery Le Barc de Bouteville in 1896 and in the Salon of the société nationale des beaux-arts (SNBA). During these exhibitions, these architects displayed uncommissioned architectural projects. This fact is important because these projects would never be built. Their styles were noticed by the great critics of the time. Their designs resulted from the will to create a new style of architecture. The architects' interest in music, poetry and literature linked them to a major artistic movement of the time: the Symbolist movement. This was a movement and not a school because no symbolist doctrine was clearly established. Garas, Guillemonat and Provensal managed to bring to life examples of their Symbolist influence through their architectural projects. In their work, they made reference to symbolist artists and appreciated art by Symbolists with whom they shared the same approach.

Drei im späten 19. und frühen 20. Jahrhundert tätige Architekten wurden als «ideistische Architekten » bezeichnet : François Garas (1866-1925), Gabriel Guillemonat (1866-1945) und Henri Provensal (1868-1934). Obwohl sie keine echte Gruppe bildeten, teilten sie anscheinend ähnliche Lebensläufe und geistige Auffassungen. Alle drei wurden an der Pariser École des Beaux-Arts ausgebildet und stellten ihre Werke 1896 in der Galerie Le Barc de Bouteville aus und dann regelmäßig im Salon de la Société nationale des Beaux-Arts (SNBA). Als Beitrag zu diesen verschiedenen Anlässen stellten sie ausschließlich architektonische Entwürfe ohne Auftraggeber aus, was umso bedeutungsvoller ist, dass die Werke nie zustande kamen. Ihre Projekte, die das Interesse der angesehenen damaligen Kritik weckten, zeugen von ihrem Willen zu einer neuen Auffassung der Architektur, die sie sozusagen neu gründen wollten. Durch ihr besonderes Interesse für Musik, Lyrik und Literatur näherten sie sich der damals bedeutsamsten künstlerischen Bewegung des Symbolismus an. Es geht um eine Bewegung, keineswegs um eine Schule, denn nie wurde eine klare symbolistische Lehre aufgestellt.Garas', Guillemonats und Provensals architektonische Entwürfe stehen für eine zu seltene Ausführung von angewandten symbolistischen Überlegungen. Sie bezogen sich auf die Künstler dieser Bewegung, mit denen sie die gleiche geistige Auffassung teilten, ebensowie auf die von den Symbolisten geschätzten Künstler.

\section{AUTEUR}

\section{MARIE-ASTRID POURCHET}

Marie-Astrid Pourchet est née en 1984. Elle est titulaire d'un master d'histoire de l'université de Paris IV suite à la rédaction d'un mémoire portant sur la cité internationale universitaire de Paris sous la direction de Jean-Pierre Chaline. Elle est également diplômée du premier cycle de l'École du Louvre. Au cours de l'année de muséologie de l'École du Louvre, elle a étudié sous la direction de Catherine Chevillot les « architectes idéistes » de la fin du XIX ${ }^{\mathrm{e}}$ siècle et du début du XX siècle. Adresse électronique : mastridpourchet@hotmail.com 